\title{
Effects of food intervention in adults with overweight or obesity
}

\begin{abstract}
Introduction: The prevalence of obesity and overweight in Portugal Has Been Increasing. Thus, it is essential to assess the nutrition consultation, promoting the effective intervention.

Objectives: To study the effect of dietary intervention on the evolution of body composition and the adoption of healthy eating habits in overweight or obese patients.

Methodology: This was a prospective study in a non-randomized sample of overweight or obese Patients of BOTH sexes who were monitored monthly for 3 months in the external consultations of nutrition of the Portimão Unit - Hospitalar Universitário center of the Algarve. The information regarding eating habits was through Obtained the 24-hour recalls. Bioimpedance Were used to evaluate body composition.

Results: The study included 38 Patients (22 women and 16 men), who had an average age of $57.8 \pm 12.7$ years. There was a stasticall antropometric diference values in the nutritional before and after $(p<0.01)$. There was a statistically significant Increase in the number of daily meals $(p<0.01)$ and the consumption of fruit and vegetables $(p<0.05)$. The Increased physical activity and the number of daily meals, fruit and vegetable consumption and water intake were not Significantly Associated with the decrease in the percentage of fat mass.
\end{abstract}

Conclusions: Targeted dietary intervention was essential in Promoting healthy eating habits.

Keywords: anthropometry, eating habits, dietary intervention, obesity
Volume 9 Issue 5 - 2019

\section{Laranjeira Natércia, Duarte Fabiana, Alves Ana \\ Dietary Service and Nutrition, University Hospital Center of Algarve, Portugal}

Correspondence: Ana Paula Moreira Carvalho Alves, Dietary Service and Nutrition, University Hospital Center of Algarve, Portugal,Tel+35191704826I, Email ana.p.alves44@gmail.com

Received:September 12, 2019 | Published: September 27, 2019

\section{Introduction}

Obesity is a major risk factor for the development of chronic diseases, such as hypertension, dislipidemia, diabetes mellitus (DM) 2 , osteoarthritis, certain cancers and cardiovascular diseases. ${ }^{1,2}$ One sustained weight loss of only $3 \%$ to $5 \%$ may produce clinically significant benefits in some cardiovascular risk factors. ${ }^{2}$ In 2016, more than 1.9 billion (39\%) of overweight adults had 650 million (13\%) of whom were obese. ${ }^{3}$ Nationally, $22 \%$ of Portuguese are obese and $34 \%$ pre-obesity. ${ }^{4}$ According to the study based on data from the National Food Survey and Physical Activity (IAN-AF) conducted from 2015 to 2016, six out of ten Portuguese are pre-obese or obese. ${ }^{4}$ Obesity is defined as a condition in which there is excess body fat. The co-morbidities are the result of excess fat and not overweight. Currently, adipose tissue has been considered a new endocrine organ that actively synthesizes a wide variety of potent bioactive signaling molecules with autocrine, paracrine and endocrine functions. There are differences in the specific site of secretion of some of the molecules produced by adipose tissue that may help explain the large pathogenicity of intra-abdominal fat, however, in general its synthesis appears to be proportional to the total number of adipócitos.O body weight and body mass index (BMI) do not reflect or body composition or fat distribution. ${ }^{5}$

The first approach after the diagnosis of a situation of excess weight or obesity should be a referral to the nutritional consultation which is a structured process, aiming a personalized intervention.
Nutritional therapy is necessarily multifocal, ie, addressed to a wide range of behaviors and using strategies based, facilitating sustainable behavioral change. It is known to its action in improving chronic disease biomarkers, and adult nutrition consultation in primary care has shown to be effective in improving diet quality, on glycemic control and weight loss. ${ }^{6}$ Important to know the effectiveness of nutrition consultation in Portugal, secondary care, where patients with obesity and excess weight that are referenced have associated co-morbidities.

\section{Goals}

Study the effect of dietary intervention in the evolution of body composition and adoption of healthy eating habits in patients with overweight or obesity.

\section{Methodology}

Prospective study, a convenience sample of patients of both genders who are overweight or obese who attended the outpatient nutrition in Portimão Unit of University Hospital Central Algarve. Constituted inclusion criteria: age less than 18 years; $\mathrm{BMI} \geq 25 \mathrm{~kg} / \mathrm{m}^{2}$; be assiduous to monthly nutrition consultations for a period of three months. The harvest of the clinical history allowed the identification of personal medical history of the patient. On the other hand, the information regarding eating habits (number of meals, consumption of fruit and vegetables and water intake) was obtained by Previous 24 hours survey The quantification of meals was carried out using 
the standardized portion sizes. Indeed, it is considered that a portion of fruit corresponds to a medium-sized piece of fruit $(160 \mathrm{~g})$ and a portion of vegetables is 2 cups

Only raw vegetables $(180 \mathrm{~g})$ or 1 lunch cup cooked vegetables $(140 \mathrm{~g}) .^{7}$ The quantification of the daily water intake was, performed by analyzing the number of glasses of water ingested $200 \mathrm{ml}$. Patients were also asked about the practice of physical exercise, which were categorized as physically inactive or active (patients physical exercise at least three times a week and at least 30 minutes per session). With regard to the anthropometric proceeded to the measurement height through a stadiometer (sensitivity $0.1 \mathrm{~cm}$ ), according to the orientation of the CEO OR General Directorate of Health (Guideline No. 017/2013 of 05.12.2013). ${ }^{8}$ The weight and BMI were obtained by scale Tanita Body Composition Analyzer TBF-300 (sensitivity $0.1 \mathrm{~kg}$ ). The assessment of the body composition (percent fat mass, lean body mass and total body water) was made by the technique of bioimpedance patients were evaluated during the morning between 9:30 and 12 hours. The patient was asked to take all metals urinate for at least 30 minutes prior to evaluation, avoiding the consumption of food and beverages to 4 hours prior to testing. The evaluation was performed with the patient at rest and with exercise to 8 hours before it was not recommended; avoid drugs on the evaluation as to cause water retention, not being in the menstrual period, not be febrile not drink alcohol within 48 hours, so as to avoid excessive consumption of tea, coffee the day before and the day before the test. The request for collaboration to patients was made by informed consent, free and clear, in accordance with the standards of the Helsinki. The study had a favorable opinion of the Ethics Committee and the Board of Directors of the Hospital Universitario Central Algarve.

The dietary intervention was appropriate to individual nutritional needs, taking into account the preferences, intolerances and food allergies. Initially it was questioned the motivation for weight loss and if the user is motivated found (action phase a) was prepared food plan with a restriction $500 \mathrm{kcal}$ total energy value (TEV) daily. Indeed, energy needs were calculated using the formulas of the Food and Nutrition Board. ${ }^{9}$ For the distribution of VET by macronutrients, assumed is a distribution of $50 \%$ carbohydrates, $20 \%$ protein and $30 \%$ lipids.

Table I Characterization of the sample, according to the evolution of body composition and dietary habits between the first and third consultations nutrition

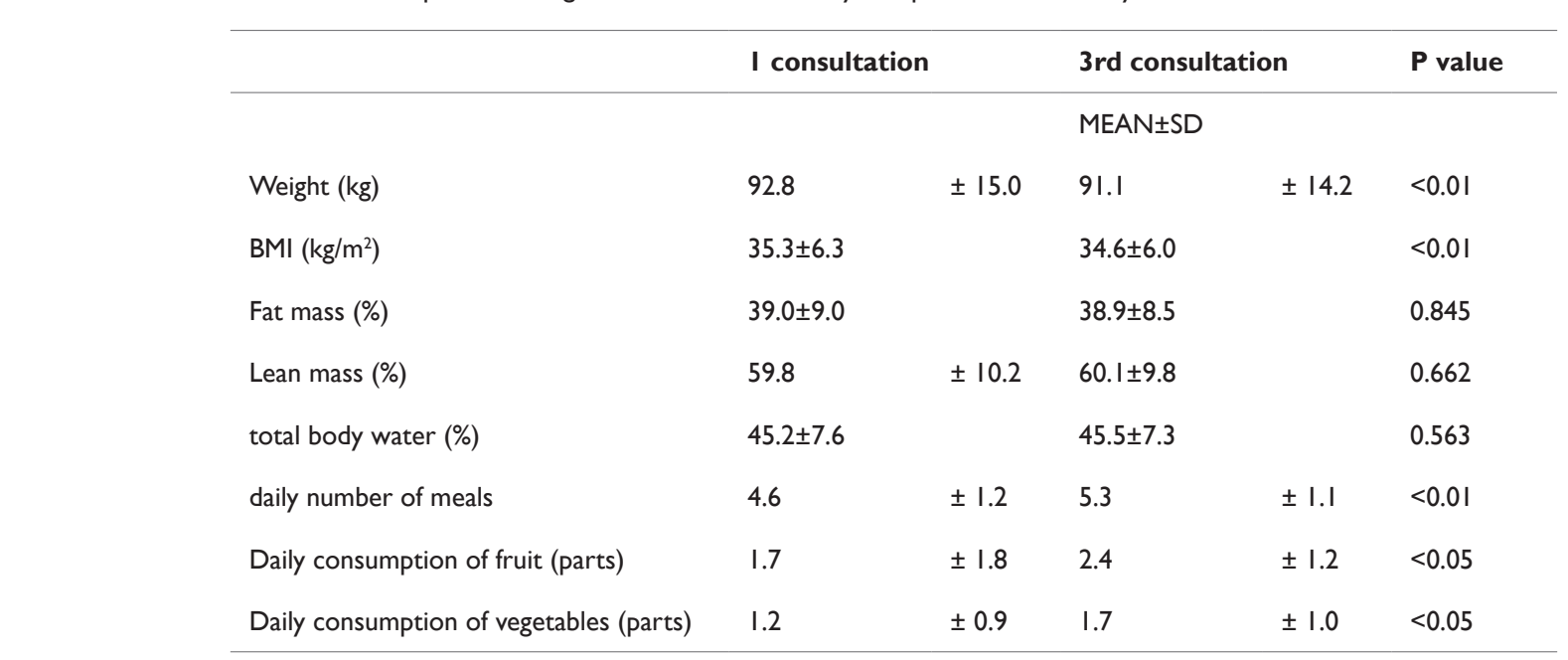

The collected data were analyzed and processed using the Statistical Package for Social Sciences, version 25. In the analysis and description of the characteristics of the sample, the quantitative variables were expressed as mean value \pm standard deviation and qualitative and frequencies. To verify the existence of significant differences in the variable before and after implementation of nutritional therapy used the paired Student's t-test. The association between variables was tested by chi-square independence for categorical variables. Assumed a significance level of $5 \%(\mathrm{p}<0.05)$.

\section{Results}

The study sample consisted of 38 individuals, of which $57.9 \%$ were female and $42.1 \%$ male. The patients were aged between 25 and 80 years and the mean age was $57.8 \pm 12.7$ years. It was found a prevalence of hypertension of $54.5 \%$ and $56.3 \%$ in females and male, respectively. Similarly, there was a greater prevalence of DM in men $(56.3 \%)$ than in women $(27.3 \%)$. We found a statistically significant difference between anthropometric values before and after dietary intervention individual $(\mathrm{p}<0.01)$. It is, therefore, a reduction in mean weight $(92.8 \pm 15.0$ vs. $91.1 \pm 14.2 \mathrm{~kg})$ and BMI $(35.3 \pm 6.3$ versus $34.6 \pm 6.0 \mathrm{~kg} / \mathrm{m}^{2}$ ) between the first and third queries nutrition, corresponding to a state of obesity and obesity Class 2 Class 1, respectively. On the other hand, there was a statistically significant increase in the number of daily meals $(\mathrm{p}<0.01)$ and number of fruit and vegetable servings consumed daily $(\mathrm{p}<0.05)$ after dietary intervention (Table 1).

There was a decrease in the percentage of fat mass over half of patients $(55.3 \%)$, although no statistically significant difference between the female and male (63.6 vs. $43.8 \%, \mathrm{p}=0.224$ ). The proportion of patients who decreased the percentage of fat mass was higher in the group of physically active patients $(\mathrm{p}=0.859)$ and group of patients that had an increased consumption of vegetables $(\mathrm{p}=0.691)$ and water intake $(p=0.847)$. Similarly, the decrease in the percentage of fat mass was not significantly associated with the number of daily meals $(p=0.744)$ and the number of servings of fruit consumed daily $(\mathrm{p}=0.154)$ (Table 2). 
Table 2 Characterization of patients showed a decrease in the percentage of fat mass, according to sex, physical activity and eating habits

\begin{tabular}{|c|c|c|c|c|}
\hline \multirow[t]{2}{*}{ Variable } & \multirow[t]{2}{*}{ Total } & \multicolumn{2}{|c|}{ Decreased\% fat mass } & \multirow[t]{2}{*}{$P$ value } \\
\hline & & $\mathrm{N}$ & $\%$ & \\
\hline Sex & & & & 0.224 \\
\hline Feminine & 22 & 14 & 63.6 & \\
\hline Male & 16 & 7 & 43.8 & \\
\hline Physical activity & & & & 0.859 \\
\hline Yes & 14 & 8 & 57.1 & \\
\hline Not & 24 & 13 & 54.2 & \\
\hline \multicolumn{2}{|c|}{ daily number of meals } & & & 0.744 \\
\hline Increased & 19 & 10 & 52.6 & \\
\hline not increased & 19 & II & 57.9 & \\
\hline \multicolumn{2}{|c|}{ Daily consumption of fruit } & & & 0.154 \\
\hline Increased & 22 & 10 & 45.5 & \\
\hline not increased & 16 & 11 & 68.8 & \\
\hline \multicolumn{2}{|c|}{ Daily consumption of vegetables } & & & 0.691 \\
\hline Increased & 17 & 10 & 58.8 & \\
\hline not increased & 21 & II & 52.4 & \\
\hline water intake & & & & 0.847 \\
\hline Increased & 23 & 13 & 56.5 & \\
\hline not increased & 15 & 8 & 53.3 & \\
\hline
\end{tabular}

\section{Discussion of results}

The nutritional therapy directed to weight reduction is recommended a gradual loss of 5 to $10 \%$ of the initial weight in a period of 6 months, which can prevent and control diseases associated with obesity. ${ }^{2,10}$ Indeed, studies have shown the effectiveness of therapeutic nutritional loss and weight maintenance. ${ }^{6,11}$ In the same way, as a result was obtained in this study a significant decrease in weight and BMI.

Nutrition consultation is a structured process that aims to promote healthy eating habits and hence improve the long-term health. ${ }^{6}$ Indeed, modification of feeding behavior is recognized as one of therapeutic approaches first line of chronic diseases such as obesity. ${ }^{6,12}$ Adequate consumption of foods rich in nutrients and low in energy, in particular fruit and vegetables, and the simultaneous decrease in intake of high energy density foods provide the energy gap. ${ }^{13,14}$ Thus, increasing the consumption of fruit and vegetables may decrease the energy density of the diet and thus promote weight loss. ${ }^{2,13,14}$ Regular physical activity is also important to increase the energy expenditure, but there was a high prevalence of physical inactivity $(63.2 \%)$ in the sample.

The World Health Organization recommends consumption of at least 400 grams of fruit and vegetables a day (equivalent to five or more servings daily). ${ }^{12}$ According to the IAN-AF 2015-2016, more than half of the Portuguese population (53\%) did not meet the aforementioned recommendation. Indeed, the Portuguese had an average daily consumption of 224 grams of fruit and 164 grams of vegetables. ${ }^{15}$ In this study, the consumption of fruit and vegetables before and after dietary intervention was also lower than that recommended, as there was an average daily consumption of fruit portions higher than vegetables. However, there was a significant change in feeding behavior, since it was found the increase in the average daily consumption of fruit and vegetables after the dietary intervention, corroborating the results obtained by other authors. ${ }^{14}$ Wagner et al., ${ }^{14}$ they evaluated the effect of food and nutritional education on the consumption of fruit and vegetables in adults overweight or obese. After 10 weeks, participants in the two intervention groups reported a significant increase in weekly frequency of average consumption of fruit and vegetables. ${ }^{14}$

The short time frame under which the dietary intervention can justify the failure to find any significant association between change in eating habits (daily number of meals, daily consumption of fruit and vegetables and water intake) and body composition. This study has certain limitations, including the limited sample size, was not carried out a control group and the short time under which the dietary intervention. The use of the survey related to the previous 24 hours was chosen as the most suitable to the time available for nutrition consultation. Always a food history as possible was requested, but as it got a low response, we chose to use this method. Knowing that cannot be performed representative of food intake and be dependent on the patient's memory. Another limitation relates to the fact that we did not consider the waist perimeter for analysis, which would have been relevant for a better characterization of the sample to the level of anthropometric parameters.

\section{Conclusions}

The targeted dietary intervention promoted to increase the number of fruit and vegetables consumed portions. In addition, nutritional therapy resulted in a reduction of anthropometric values (weight and $\mathrm{BMI})$.

\section{Acknowledgements}

None.

\section{Conflicts of interest}

The author declares that there is no conflict of interest.

\section{Funding details}

None.

\section{References}

1. World Health Organization. Obesity: Preventing and managing the global epidemic: report of a WHO consultation (WHO technical report series, 894). Geneva; 2000.

2. Jensen MD, Ryan DH, Apovian CM, et al. 2013 AHA / ACC / TOS Guideline for the Management of Overweight and Obesity in Adults. Circulation. 2014;129(25 suppl 2):S102-138.

3. World Health Organization. Obesity and overweight. 2018.

4. Oliveira J Araujo, Severus M, Belt D, et al. Prevalence of general and abdominal obesity in Portugal. Comprehensive results from the National Food, Nutrition and Physical Activity Survey. 2015-2016. BMC Public Health. 2018;18(1):614.

5. Prentice AM, Jebb SA. Beyond body mass index. Obes Rev. 2001;2(3):141-147. 
6. Mitchell LJ, Ball LE, Ross LJ, et al. Dietetic Consultations in Effectiveness of Primary Health Care: A Systematic Review of Randomized Controlled Trials. J Nutr Diet Acad. 2017;117(12):1941-1962.

7. Faculty of Nutrition and Food of the University of Porto. Guide: The Food at the wheel. Consumer Institute; 2003.

8. Directorate General of Health Guidance. Anthropometric Assessment in Adult. 2013.

9. Institute of Medicine. Dietary Reference Intakes for Energy, Carbohydrate, Fiber, Fat, Fatty Acids, Cholesterol, Protein, and Amino Acids. Washington, DC: National Academies Press; 2005.

10. Kushner RF, DH Ryan. Lifestyle Assessment and Management of Patients with Obesity. JAMA. 2014;312(9):943-952.

11. Nurkkala M, K Kaikkonen, Vanhala ML, et al. Lifestyle intervention has a beneficial effect on eating behavior and long-term weight loss in obese adults. Eat Behav. 2015;18:179-185.
12. World Health Organization. Diet, nutrition and the prevention of chronic diseases: report of a joint WHO/FAO expert consultation (WHO Technical Report Series, 916). Geneva; 2003.

13. Rolls BJ. What is the role in control of weight management portion? Int $J$ Obes. 2014;38(S1):S1-8.

14. Wagner MG, Rhee Y, Honrath K, et al. Effective education in Nutrition Increasing fruit and vegetable consumption among overweight and obese adults. Appetite. 2016;100:94-101.

15. Lopes C, D Torres, Oliveira, et al. National and physical activity nutrition survey (IAN-AF) 2015-16. University of Porto; 2017. 